\title{
The two CD95 apoptosis signaling pathways may be a way of cells to respond to different amounts and/or forms of CD95 ligand produced in different tissues
}

Dear Editor,

We have recently identified two CD95 (APO-1/Fas) signaling pathways which are used by different cell types. ${ }^{1}$ Type I cells show optimal formation of the CD95 death-inducing signaling complex (DISC) initiating a direct caspase cascade independent of mitochondrial changes during apoptosis. Therefore, apoptosis signaling in these cells cannot be blocked by antiapoptotic Bcl-2 family members. In contrast, in type II cells DISC formation is strongly reduced. Low amounts of caspase8 activated at the DISC are not sufficient to induce a direct caspase cascade. Thus, type II cells depend on an amplification step. Upon cleavage of the pro-apoptotic Bcl-2 family member Bid, truncated Bid translocates to the mitochondria and induces cytochrome $c$ release and loss of mitochondrial transmembrane potential. ${ }^{2-4}$ In the cytosol, cytochrome $c$ binds Apaf- 1 and dATP forming the apoptosome. Subsequently caspase-9 is recruited to the apoptosome and activated initiating a caspase cascade downstream of mitochondria. ${ }^{5,6}$ These studies were performed in cell lines using the agonistic monoclonal anti-CD95 antibody anti-APO1 to induce apoptosis. Further studies using CD95 ligand (L) or primary human $\mathrm{T}$ cells confirmed this model. ${ }^{7-9}$

Recently Huang et al reported that they do not find evidence for different pathways in CD95 signaling. ${ }^{10}$ However, there are a number of problems attached with some experimental approaches used by these authors and with the citations and interpretations of the data which we like to address in the following:

(1) Huang et a/ ${ }^{10}$ suggest that membrane bound CD95L ( $\mathrm{mCD} 95 \mathrm{~L})$ is the physiologically active form of the ligand. This may be true for certain tissues but soluble CD95L (sCD95L) is also cytotoxic on certain cells. Jurkat $T$ cells cultured as single cells undergo activation induced cell death, due to autocrine suicide most likely involving SCD95L. ${ }^{11}$ In addition a supernatant of these cells was shown to kill CD95 sensitive cells, albeit weakly..$^{11}$ Moreover Huang et al ${ }^{10}$ used CD95L overexpressing cell lines as effector cells. Whether these CD95L transfectants reflect the expression level of mCD95L expressing cells and mimic CD95L activity in vivo remains to be shown. In addition, the role of $\mathrm{mCD} 95 \mathrm{~L}$ vs SCD95L in apoptosis induction still has to be elucidated in vivo.

(2) Huang et $\mathrm{al}^{10}$ state that trimerization of the CD95L is not sufficient to kill cells and that aggregation of CD95 is required to give it its full cytotoxic activity. The leucine zipper tagged CD95L (LZ-CD95L) used in our study specifically trimerizes and is highly active. We have compared the activity of LZ-CD95L and Flag-tagged and crosslinked CD95L side by side and found them to be similarly active. Which of these ligands when injected into a mouse resembles more the physiological situation? Neither of them. Our view is that such experiments should not be done with recombinant human ligand but with endogenous murine ligand. A system to do that is available $^{12}$ and experiments to test the murine CD95L are under way.

(3) Huang et $a l^{10}$ state that cells from mice deficient of either caspase-9 or Apaf-1 are normally sensitive to CD95 mediated apoptosis. In the original characterization of the Apaf- 1 knock out mice one group only tested activated peripheral $\mathrm{T}$ cells and thymocytes and found the CD95 pathway to be independent of the mitochondrial pathway. ${ }^{13}$ This is consistent with our data since we have identified these tissues as type I. However, another group tested embryonic fibroblasts and found a $70 \%$ reduction of sensitivity to CD95 mediated apoptosis in these cells. ${ }^{14}$ This report, therefore, also suggested the presence of two CD95 pathways. The case of the caspase- $9^{-1-}$ mice is similar to the Apaf-1 $1^{-1-}$ mice since also in the two reports characterizing these mice only activated $T$ cells (splenocytes) and/or thymocytes were tested. ${ }^{15,16}$ The phenotype of the Apaf- 1 and caspase-9 knock out mice is, therefore, not in contradiction to our proposed models of two CD95 pathways.

(4) Huang et $a /^{10}$ state that CD95L used in their studies induces extensive receptor aggregation. However, they did not provide any data on aggregation of CD95. We have found that upon stimulation of cell lines or primary human $T$ cells with the agonistic monoclonal anti-CD95 antibody, anti-APO-1, CD95 forms trimers and oligomers. Both, trimers and oligomers, correlate with DISC formation (unpublished data).

(5) Huang et $a l^{10}$ also state that upon stimulation with crosslinked CD95L they observed no difference in FADD and caspase-8 recruitment to the plasma membrane in type I and type II cells. However, their way of DISC analysis is technically insufficient. They did not purify plasma membranes (at which the DISC is located) but only a crude membrane fraction. ${ }^{10}$ Nonspecific aggregation of FADD and caspase-8 during the preparation cannot be excluded in this experimental procedure. We believe that DISC formation can only convincingly be demonstrated by immunoprecipitating cell surface death receptors 
stimulated by either agonistic antibodies or, alternatively, via the DISC-inducing death ligands as has been shown for CD95L and TRAIL. 7,17,18,19,20

(6) Huang et $a l^{10}$ did not detect any protection against CD95-mediated apoptosis by $\mathrm{Bcl}-2$ or Bcl- $\mathrm{x}_{\mathrm{L}}$. However, they did not perform titrations of the apoptosis inducing reagents. We observed complete or at least partial inhibition of CD95-mediated apoptosis in Bcl-2 and $\mathrm{Bcl}-\mathrm{x}_{\mathrm{L}}$ expressing type II cells depending on the expression levels of $\mathrm{Bcl}-2$ and $\mathrm{Bcl}-\mathrm{x}_{\mathrm{L}} \cdot{ }^{1,7}$ Resistance or sensitivity towards CD95-mediated apoptosis in type II cells, however, almost certainly depends on the ratio of the amounts of CD95L and the expression of $\mathrm{Bcl}-2$. Furthermore, as pointed out above, these experiments should be done in a model that involves the endogenous murine CD95L. Nothing is known about the actual amounts of either sCD95L or mCD95L in different tissues. The role of CD95 on hepatocytes and the natural source of CD95L in these tissues is not even known. It is highly likely that physiological levels of CD95L may vary in different tissues. It is therefore conceivable that lower levels of mCD95L or high levels of sCD95L affect type I and type II cells differently. The type I type II distinction may be an adaptation of certain cells to different amounts or forms of CD95L. In addition, it is completely unknown in which tissues soluble and membrane bound CD95L is active. Since in the mouse $\mathrm{SCD} 95 \mathrm{~L}$ has not been found whereas in humans AICD of peripheral $T$ cells is likely mediated by SCD95L the approach used by Huang et $a l,{ }^{10}$ namely, to inject FLAG tagged soluble human CD95L into mice is highly questionable and it is not clear whether this form of the ligand resembles endogenous murine mCD95L as claimed by the authors.

(7) Huang et $\mathrm{al}^{10}$ find it remarkable that $\mathrm{Bcl}-2$ transgenic mice or BID deficient mice were only partially protected from CD95 mediated apoptosis when injected with intermediate doses of anti-CD95 antibodies whereas cells from either FADD or caspase-8 knock out mice were completely resistant. That is not surprising since FADD and caspase- 8 are essential components of both CD95 pathways whereas blocking of only the mitochondrial pathway leaves the mitochondria independent pathway intact. It may be the relative contribution of both pathways in a given tissue that determines the sensitivity to CD95 mediated apoptosis. We did not find and never claimed to have found a complete block of the CD95 pathway in type II cells overexpressing Bcl-2 or Bcl- $\mathrm{x}_{\mathrm{L}}$. At very high concentrations of either anti-CD95 or LZCD95L Bcl-2 overexpressing Jurkat cells are not protected from death. ${ }^{1,7}$ However, we find it remarkable that again the degree of protection by $\mathrm{Bcl}-2$ was similar when we compared anti-CD95mAbs and CD95L.

(8) Huang et $a l^{10}$ wrote in their publication in PNAS that 'experiments with primary or cultured lymphoid cells showed that $\mathrm{Bcl}-2$ or $\mathrm{Bcl}-\mathrm{x}_{\mathrm{L}}$ overexpression had no impact on cell death induced by anti-CD95 antibodies even though they efficiently inhibited apoptosis induced by other cytotoxic treatments'. This statement completely ignores a report by Schneider et al, ${ }^{21}$ who found that $\mathrm{BCl}-\mathrm{x}_{\mathrm{L}}$ protects primary $\mathrm{B}$ cells against CD95 mediated apoptosis. These data were not generated with an anti-CD95 antibody but with mCD95L expressing Th1 effector cells ${ }^{19}$ demonstrating that $\mathrm{Bcl}-\mathrm{x}_{\mathrm{L}}$ can protect certain cells from CD95 mediated apoptosis also when apoptosis is induced by the physiological ligand. B cells are, therefore, type II cells.

(9) Even if the differences between type I and type II cells could only be detected with an anti-CD95 antibody as compared to the cognate ligand the antibody would still be a good analytical tool to detect differences between the two cell types. We have recently found that CD95 expressed on type I and type II cells is structurally different (unpublished observation) and we are currently studying this phenomenon. By comparing type I and type II cells Huang et $a l^{10}$ therefore confirmed our data that CD95 expressed on type I and type II cells is different since they also found that CD95 on only the type II cells requires crosslinking of the anti-CD95 antibody to be fully active. Their finding is consistent with our data (unpublished observation) and confirm our initial observation that the receptor expressed on type and type II cells is different. However, despite acknowledging the differences in stimulation of type I and type II cells Huang et al ${ }^{10}$ argued that these differences might solely be due to different expression levels of CD95 on these two cell types. ${ }^{10}$ It is correct that both type II cells Jurkat and CEM express slightly less CD95 on their surface than the tested type I cells SKW6.4 and H9. However, we also identified and characterized a type II cell with higher CD95 expression than any of the type I cells. ${ }^{9}$ The pre B cell line BoeR can be sensitized to undergo CD95 mediated apoptosis by treating it with cycloheximide. However, this treatment does not change expression of CD95 or its inability to form a DISC. Consistent with the findings in other type II cells crosslinking of anti-CD95 mAb with protein A strongly enhances its cytotoxic activity (unpublished data).

(10) Finally, differences between type I and type II cells are not even limited to the way CD95 is triggered at the cell surface. We also found that only our prototype type II cells are sensitive to induction of apoptosis by the ceramide analog C2-ceramide whereas all type II cells were completely resistant. ${ }^{9}$ Differences between type I and type II cells may therefore exist on various levels not just at the level of the CD95 receptor.

Taken together, the differences observed in experiments by Huang et $a l^{10}$ and our laboratories may be explained by technical differences in experimentation. In addition, extensive crosslinking of CD95L, be it physiological or not, may blur type I and type II differences. 
I Schmitz ${ }^{1}$, H Walczak ${ }^{1}$, PH Krammer ${ }^{1}$ and ME Peter ${ }^{2}$

${ }^{1}$ Tumorimmunology Program, German Cancer Research Center, Im Neuenheimer Feld 280, 62120 Heidelberg, Germany.

E-mail: P.Krammer@dkfz-heidelberg.de; ${ }^{2}$ Ben May Institute for Cancer Research, University of Chicago, 924 E 57th Street, Chicago, Illinois, IL 60637, USA. E-mail: MPeter@ben-may.bsd.uchicago.edu

1. Scaffidi C et al (1998) EMBO J. 17: 1675

2. Li H et al (1998) Cell 94: 491

3. Luo X et al (1998) Cell 94: 481

4. Gross A et al (1999) J. Biol. Chem. 274: 1156

5. Zou H et al (1997) Cell 90: 405
6. Li P et al (1997) Cell 91: 479

7. Schmitz I et al (1999) Cell Death Diff. 6: 821

8 Peter ME et al (1997) Eur. J. Immunol. 27: 1207

9. Scaffidi C et al (1999) J. Biol. Chem. 274: 22532

10. Huang DCS et al (1999) PNAS 96(26): 14871

11. Dhein J et al (1995) Nature 373: 438

12. Seino KL et al (1997) Gastroenterology 113: 1315

13. Yoshida $\mathrm{H}$ et al (1998) Cell 94: 739

14. Cecconi $\mathrm{F}$ et al (1998) Cell 94: 727

15. Hakem R et al (1998) Cell 94: 339

16. Kuida K et al (1998) Cell 94: 325

17. Kischkel FC et al (1995) EMBO J. 14: 5579

18. Bodmer JL et al (2000) Nat. Cell Biol. 2: 24

19. Sprick MR et al (2000) Immunity (in press)

20. Kirschkel FC et al (2000) Immunity (in press)

21. Schneider TJ et al (1997) J. Immunol. 159: 4834 